\title{
Forecasting Area, Yield and Production of Groundnut Crop: A Comparative Study of Coastal Andhra and Ceded Regions Using- R
}

\author{
Ananda Kumar Ginka, Dhanunjaya Sunkara, Mohammed Akhtar
}

\begin{abstract}
This comparative study has been carried out to discuss all investors are excited to know about the trend of groundnut area, yield and production in Coastal Andhra and Ceded Regions (Seemandhra). Now it has 13 districts during 2003-2004 to 2018 (15 years of data) for present comparative analysis of groundnut crop production in Coastal Andhra and Ceded were collected and presented. Based on results collected some conclusions are made about the forecast production of groundnut crop by using ARIMA with Rsoftware.
\end{abstract}

Index Terms - Area, yield, production, Groundnut, ARIMA, R software.

\section{INTRODUCTION}

Time is one of the most important factors on which our business and real-life dependent situations. But

now a day's technology has helped us manage the time with continuous innovations taking place in all aspects

of our lives. Time series modelling is a dynamic research area which has attracted the attention of researcher's community over last few decades. This model is used to generate future values for the series, i.e to make forecasts. Forecasting lays a ground for reducing the risk in all decision making because many of decisions need to be made under uncertainty.

India is an agricultural country. The Economic progress and standard of living of people directly or indirectly is determined by agriculture. Indian economy mainly depends on agriculture as majority of the population depends on agricultural and allied activities for seeking out their livelihood. Groundnut is grown throughout the tropics and its cultivation is extended to the subtropical countries lying between $45^{\circ}$ North and $35^{\circ}$ South and up to an altitude of 1,000 meters. The total amount of rainfall required for presuming operations (preparatory) is $100 \mathrm{~mm}$, for sowing it is $150 \mathrm{~mm}$ and for flowering and pod development an evenly

Manuscript revised on December 25, 2019 and published on January 10,2019

Ananda Kumar Ginka, currently working as a Lecturer in Statistics in department of statistics, Sri Srinivasa Degree college, Madanapalle, Chittoor District, Andhra Pradesh, India.

Dhanunjaya Sunkara, M.Sc.,in Statistics and Pursuing Ph.D, Research Scholar, Department Of Statistics, Sri Krishnadevaraya University, Anantapuramu, Andhra Pradesh, India.

Mohammed Akhtar, working as a Professor and Head, Department of Statistics, Sri Krishnadevaraya university, Anantapuramu, Andhra Pradesh, India. distributed rainfall of $400-500 \mathrm{~mm}$ is required, Madhusudana , $B$ et al( 2013)[1]. Coastal Andhra is located in the eastern region of the state of Andhrapradesh on Coromandel Coast and comprises nine districts. In Andhra Pradesh groundnut is grown majorly in Srikakulam and Vishakapatnam districts of Coastal Andhra Region.

Ceded Districts is name of an area in the Deccan, India that was 'Ceded' to the British East India Company by the Nizam in 1800. The name was the British Raj, even though the denomination had no official weight for legal or administrative purposes. Rayalaseema, meaning 'rocky region', Especially, groundnut is the only important commercial crop in the drought prone district of Anantapuramu in Rayalaseema region of Andhra Pradesh. So, the district headquarter of Anantapuramu is called as 'Groundnut City'. Groundnut is an important protein supplement for cattle and poultry rations. It is also consumed as confectionery product. The cake can be used for manufacturing artificial fibre. The haulms are fed to livestock.

Crop area estimation and forecasting of crop yield are an essential procedure in supporting policy decision regarding land use allocation, food security and environmental issues. Statistical techniques able to provide crop forecast with reasonable precessions well in advanced. Various approaches have been used for forecasting such agricultural systems. Concentration have been given on the uni-variate time series Auto Regressive Integrated Moving Average (ARIMA) MODELS, which are primarily due to World of Box and Jenkins (1970). Among the stochastic time series models ARIMA types are powerful and popular as they can successfully describe the observed data and can make forecast with minimum forecast error. These types of models are very difficult to identify and estimate.

\section{LITERATURE REVIEW}

Muhammad Iqbal Ch et al.(2016) for forecasting of wheat production: A comparative study of Pakistan and India [2], Similar studies have been done by Rachana et al. (2010) for forecasting pigeon pea production in India by using ARIMA Modelling [3], N.M.F. Rahman et al. ( 2010)for forecasting of Boro rice production in Bangladesh [4], Najeeb Iqbal et al. (2005) for forecasting wheat area and production in Pakistan [5], M.K Debnath et al. (2013)for forecasting Area, production, and Yield of Cotton in India using ARIMA Model [6], M. Hemavathi et al.(2018) ARIMA Model for Forecasting of Area, Production and productivity of Rice and Its Growth Status in Thanjavur District of TamilNadu, India[7], P.K. Sahu et al.(2015) for modelling and forecasting of area, production, yield and total 
seeds of Rice and Wheat in SAARC Countries and the World towards Food Security[8], Mohammed Amir Hamjah et al.(2014) for Rice Production Forecasting in Bangladesh: An Application of Box-Jenkins ARIMA Model[9], Muhammad et al(1992) conducted an empirical study of modelling and forecasting time series data of rice production in Pakistan [10], Niaz Md. Farhat Rahman et al.(2013), Modelling for Growth and Forecasting of pulse production in Bangladesh [11], Vishwajith K..P et al.(2014), Timeseries Modeling and forecasting of pulses production in India[12], Ashwin Darekar et al.(2017), Forecasting oilseeds prices in India: Case of Groundnut [13] , Bhola Nath et al.(2018) DS , Forecasting Wheat production in India: An ARIMA modelling approach [14], Pant, D.C. and Pradeep Pal, et al.(2004), Comparative Economics of Agro-processing units for Groundnut in Southern Rajasthan [15], Ap Patel, G.N., and N.L. Agarwal et al. (1993), Price Behaviour of Groundnut in Gujarat [16], Mohammad Mayazzem Hossain(2017), Comparision of ARIMA and Neural Net Work Model to forecast the jute Production in Bangladesh, Jahingir Nagar University Journal of Science, [17] , also use the ARIMA Model . The study is to identify the best ARIMA model, which is for fitting and forecasting of Groundnut Area, Yield, Production in Ceded region respectively. Conclusions are drawn and found the forecasting for the future. The R-Software is used to analyse and graphical representation of the results.

R- software: $\mathrm{R}$ is a commonly used free Statistics software. $\mathrm{R}$ allows you to carry out statistical analyses in an interactive mode, as well as allowing simple programming. The Rlanguage is widely used among statistician and data miners for developing statistical software and data analysis. Although $\mathrm{R}$ has a command line interface, there are several graphical user interfaces, such as $\mathrm{R}$ studio, an integrated development environment. $\mathrm{R}$ is a programming language and environment commonly used in statistical computing, data analytics and scientific research. It is one of the most popular languages used by statisticians, data analysts, researchers and marketers to retrieve, clean, analyze, visualize and present data.

\section{MATERIALS AND METHODS}

\section{A. Data collection:}

The study has utilized secondary source of data. The time series data on yearly kharif and Rabi seasons totals area, yield and production of groundnut crop from 2003-2004 to 2017-2018 of 15 years data required for the study was collected from the DIRECTORATE OF ECONOMICS AND STATISTICS, HYDERABAD. The 15 years of comparative data of groundnut producing ceded districts viz., Anantapuramu, Kurnool, cuddapah, chittoor districts of Andhra Pradesh and Coastal Andhra Andhra districts viz., Srikakulam, Vizianagaram, Visakhapatnam, East Godavari, West Godavari,Krishna, Guntur, Prakasam, and Nellore districts. Coastal Andhra borders Rayalaseema regions of the state and the states of Telangana, Odisha. The presence of the Krishna River Godavari River and Penna River makes the area fertile for irrigation.

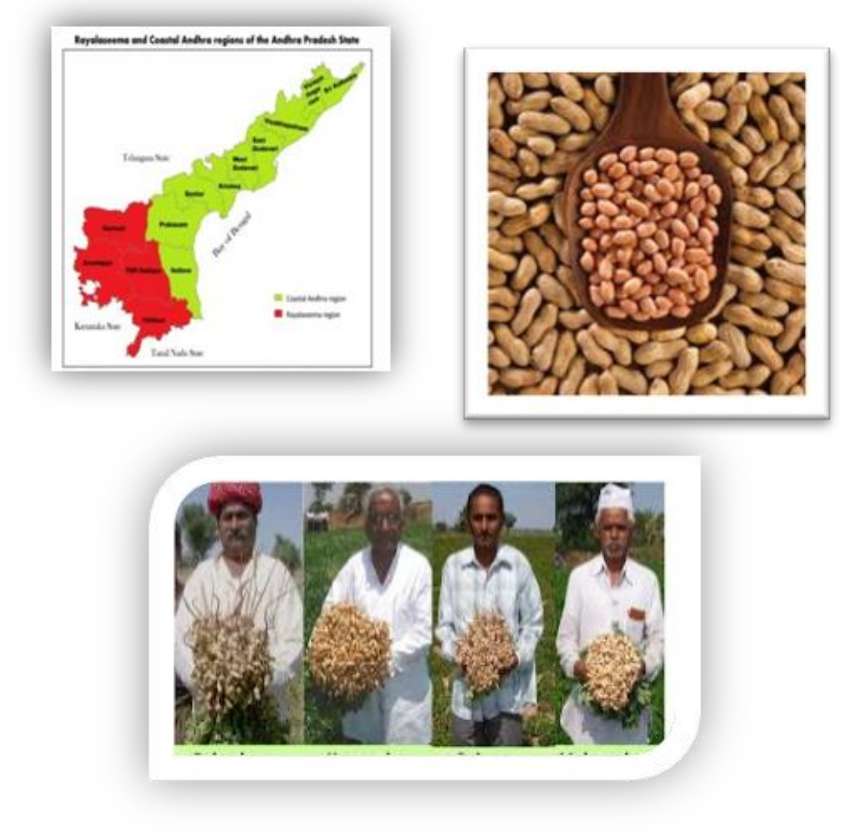

Fig:1 Area, Yield and Production of Groundnut Crop in Ceded and Coastal Andhra Regions

\section{B. Auto Regressive Integrated Moving Average (ARIMA) model (Box-Jenkins model):}

One of the most popular and frequently used stochastic time series models is the Auto Regressive Integrated Moving Average (ARIMA) model was introduced by Box and Jenkins. The basic assumption made to implement this model is that considered time series is linear and follows a particular known statistical distribution, such as the Normal Distribution. ARIMA model has subclasses of other models, such as Auto Regressive (AR), Moving Average (MA) and Auto Regressive Moving Average (ARMA) models. For seasonal time series forecasting, Box and Jenkins had proposed a quite successful variation of ARIMA model, viz. the Seasonal ARIMA (SARIMA). The popularity of the ARIMA model is mainly is due to its flexibility to represent several varieties of time series with simplicity as well as the associated Box-Jenkins(1994) [5] methodology for the optimal model building process.

The term ARIMA stands for "Auto-Regressive Integrated Moving Average." Lags of the differenced series appearing in the forecasting equation are called "auto-regressive" terms, lags of the forecast errors are called "moving average" terms, and a time series which needs to be differenced to be made stationary is said to be an "integrated" version of a stationary series. Random-walk and random-trend models, autoregressive models, and exponential smoothing models (i.e., exponential weighted moving averages) are all special cases of ARIMA models. A non-seasonal ARIMA model is classified as an "ARIMA (p, d, q)" model, where $p$ is the number of autoregressive terms, $d$ is the number of non-seasonal differences, and $\mathrm{q}$ is the number of lagged forecast errors in the prediction equation. The Box-Jenkins methodology seeks to transform any time series data to be stationary; and then apply the stationary process for 
forecasting by using past uni-variate time series process for future forecast with a host of selection and diagnostic tools.

\section{1) Model Identification}

This stage involves the specification of the correct order of ARIMA model by determining the appropriate order of the $\mathrm{AR}, \mathrm{MA}$ and the integrated parts or the differencing order. The major tools in the identification process are the (sample) autocorrelation function and partial autocorrelation function. The identification approach is basically designed for both stationary and non-stationary processes. Spikes represent in the line at various lags in the plot with length equal to magnitude of autocorrelations and these spikes distinguish the identification of a stationary and non-stationary process. The main objective in fitting ARIMA model is to identify the stochastic process of the time series and its stationarity counterpart. The main objective in fitting ARIMA models is to identify the stochastic process of the time series and predict the future values accurately. Ansari and Ahmad (2001)[18] worked with application of ARIMA modelling and co-integration analysis on time series of tea price. Different stages in forecasting model are given below. Identification: A good starting point for time series analysis is a graphical plot of the data. It helps to identify the presence of trends. Before estimating the parameters $\mathrm{p}$ and $\mathrm{q}$ of the model, the data are not examined to decide about the model which best explains the data. This is done by examining the sample ACF, and PACF. Both ACF and PACF are used as the aid in the identification of appropriate models. There are several ways of determining the order type of process, but still there was no exact procedure for identifying the model.

\section{2) Model Estimating the parameters}

After tentatively identifying the suitable model is not "estimating a second time series", it is filtering it. The function accuracy gives multiple measures of accuracy of the model fit, ME(mean error), RMSE(root mean squared error), MAE(mean absolute error), MPE(mean percentage error), MAPE(mean absolute percentage error), MASE(mean absolute scaled error), And ACF (auto correlation function) It is up to you to decide, based on the accuracy measures, whether you consider this a good fit or not. For example, mean percentage error of nearly $-70 \%$ does not look good to me in general, but that may depend on what your series are and how much predictability you may realistically expect. It is often a good idea to plot the original series and the fitted values, and also model residuals. You may occasionally learn more from the plot than from the few summarizing measures such as the ones given by the accuracy function. Depending on the ACF and PACF of these sequence plots a model is run with appropriate software (R-Software). The best fitting model must also have few parameters as much as possible alongside best statistics of the model according to the information selection criteria.

\section{3) Model Diagnostic Checking}

After having estimated the parameters of a tentatively identify ARIMA model, it is necessary to do diagnostic checking to verify that the model is adequate. Examining ACF And PACF considered random when all their ACF and PACF considered random when all their ACF were within the limits. Model checking in time series can be done by looking at the residuals. Traditionally the residuals given by Residuals $=$ observed values - fitted values. These results should be normally distributed with zero mean, uncorrelated, and should have minimum variance or dispersion, if indeed a model fits the well. That is model validation usually consist of plotting residuals overtime to verify the validation.

\section{4) Model Forecasting}

After satisfying about the adequacy of the fitted model, it can be used for forecasting future values. This was done with the help of R-Software.

\section{RESUlt AND Discussions}

Analysis of Time series data regarding agricultural oriented groundnut crop area, yield and production using $\mathrm{R}$ software tabulated along with necessary graphical presentations mentioned below, Groundnut is an important protein supplement for cattle and poultry rations. It is also consumed as confectionery product. The cake can be used for manufacturing artificial fibre. The haulms are fed to live stock. Groundnut shell is used as fuel for manufacturing coarse boards. Cork substitutes. Groundnut is also valued as a rotation crop. Being a legume with root nodules, it can synthesize with atmospheric nitrogen and thereby improve soil fertility. All investors are timely cautious about crop production with updated technology.

Table-1 Area, Yield and Production Of Groundnut Crop In Ceded And Coastal Andhra Regions

\begin{tabular}{|c|l|l|l|l|l|l|l|}
\hline \multicolumn{2}{|l|}{ CEDED REGION } & \multicolumn{3}{l|}{ COASTAL ANDHRA REGION } \\
\hline & YEAR & $\begin{array}{l}\text { Area } \\
\text { (in } \\
\text { 000'ha.) }\end{array}$ & $\begin{array}{l}\text { Yield } \\
\text { (in } \\
\text { Kg/ha.) }\end{array}$ & $\begin{array}{l}\text { Prod. } \\
\text { (in } \\
\text { 000'tones) }\end{array}$ & $\begin{array}{l}\text { Area (in } \\
\text { 000'ha.) }\end{array}$ & $\begin{array}{l}\text { Yield } \\
\text { (in } \\
\text { Kg/ha.) }\end{array}$ & $\begin{array}{l}\text { Prod. } \\
\text { (in } \\
\text { 000'tones) }\end{array}$ \\
\hline 1 & $2003-2004$ & 1164 & 2664 & 603 & $\mathbf{1 3 4}$ & $\mathbf{1 2 1 2 2}$ & $\mathbf{1 5 9}$ \\
\hline 2 & $2004-2005$ & 1511 & 3455 & 1267 & $\mathbf{1 3 6}$ & $\mathbf{1 6 2 4 9}$ & $\mathbf{1 7 5}$ \\
\hline 3 & $2005-2006$ & 1554 & 2800 & 924 & $\mathbf{1 2 3}$ & $\mathbf{1 8 4 8 9}$ & $\mathbf{1 8 6}$ \\
\hline 4 & $2006-2007$ & 1041 & 2522 & 366 & $\mathbf{1 1 4}$ & $\mathbf{1 7 0 5 9}$ & $\mathbf{1 5 7}$ \\
\hline 5 & $2007-2008$ & 1474 & 6278 & 2076 & $\mathbf{1 1 5}$ & $\mathbf{1 6 2 2 1}$ & $\mathbf{1 8 3}$ \\
\hline 6 & $2008-2009$ & 1447 & 1978 & 471 & $\mathbf{1 1 8}$ & $\mathbf{1 7 2 9 0}$ & $\mathbf{1 8 8}$ \\
\hline
\end{tabular}


International Journal of Research in Advent Technology, Vol.7, No.11, November 2019 E-ISSN: 2321-9637

Available online at www.ijrat.org

\begin{tabular}{|c|l|l|l|l|l|l|l|}
\cline { 3 - 7 } 7 & $2009-2010$ & 991 & 2588 & 493 & $\mathbf{9 6}$ & $\mathbf{1 8 3 1 6}$ & $\mathbf{1 5 4}$ \\
\hline 8 & $2010-2011$ & 136 & 3466 & 962 & $\mathbf{9 1}$ & $\mathbf{1 6 8 5 4}$ & $\mathbf{1 4 3}$ \\
\hline 9 & $2011-2012$ & 1058 & 2681 & 444 & $\mathbf{7 7}$ & $\mathbf{1 8 0 9 8}$ & $\mathbf{1 3 8}$ \\
\hline 10 & $2012-2013$ & 1089 & 2899 & 635 & $\mathbf{7 0}$ & $\mathbf{1 8 7 9 4}$ & $\mathbf{1 4 3}$ \\
\hline 11 & $2013-2014$ & 1111 & 3933 & 739 & $\mathbf{4 2}$ & $\mathbf{2 3 2 3 4}$ & $\mathbf{1 0 6}$ \\
\hline 12 & $2014-2015$ & 832 & 2774 & 391 & $\mathbf{4 2}$ & $\mathbf{2 3 2 7 8}$ & $\mathbf{1 0 2}$ \\
\hline 13 & $2015-2016$ & 732 & 4785 & 694 & $\mathbf{6 6}$ & $\mathbf{2 0 2 1 6}$ & $\mathbf{1 4 2}$ \\
\hline 14 & $2016-2017$ & 968 & 3112 & 485 & $\mathbf{4 6}$ & $\mathbf{2 3 7 6 4}$ & $\mathbf{1 1 7}$ \\
\hline 15 & $2017-2018$ & 697 & 6243 & 942 & $\mathbf{3 7}$ & $\mathbf{2 4 5 1 9}$ & $\mathbf{1 0 6}$ \\
\hline & TOTAL & 15805 & 52178 & 11492 & $\mathbf{1 3 0 7}$ & $\mathbf{2 8 4 5 0 3}$ & $\mathbf{2 1 9 9}$ \\
\hline
\end{tabular}
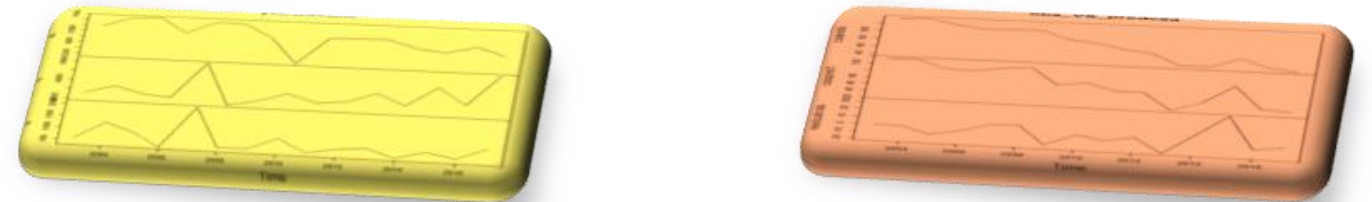

Fig:2 Area, yield, Production of Ceded Region Fig:3 Area, yield, Production of Coastal andhra Region ACF, PACF plots are analysed to check stationarity of data upto15 ( 0 to 14) lags as shown below:

Fig:4 AREA- ACF(CEDED REGION)

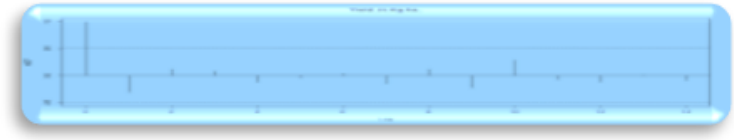

Fig:6 Yield- ACF(CEDED REGION)

Fig :8 Production -ACF(CEDED REGION)

Fig:10 Area-ACF(Coastal Region)

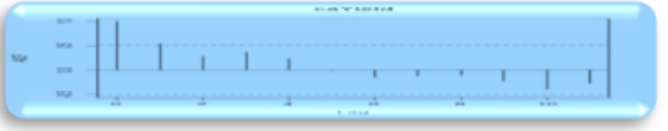

Fig:12 YIELD-ACF(Coastal Region)

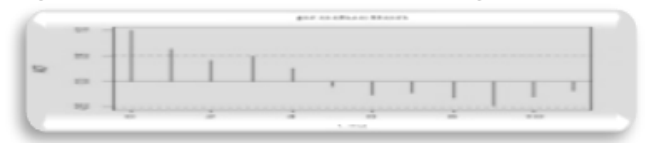

Fig:14 prod-ACF(Coastal Region)

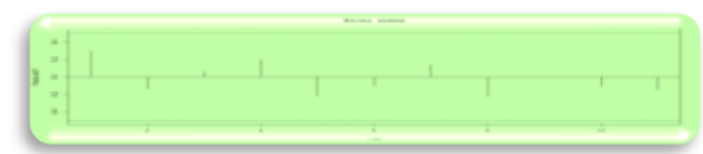

Fig:5 AREA- PACF(CEDED REGION

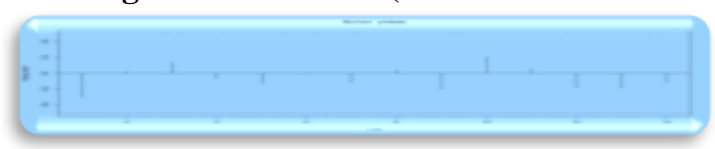

Fig: 7 YIELD - PACF(CEDED REGION)

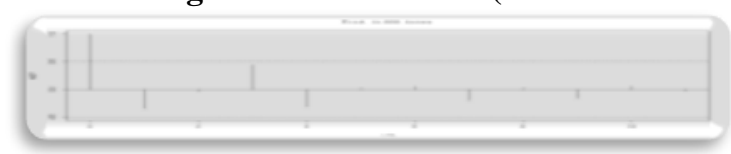

Fig:9 PRODUCTION- PACF(CEDED REGION)

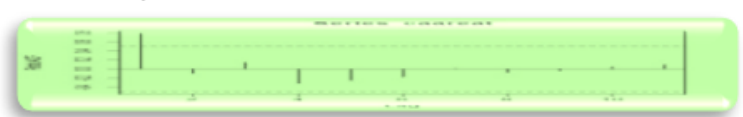

Fig:11Area-PACF(Coastal Region)

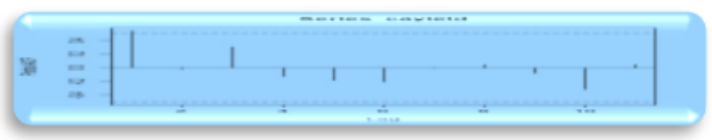

Fig:13 YIELD-ACF(Coastal Region)

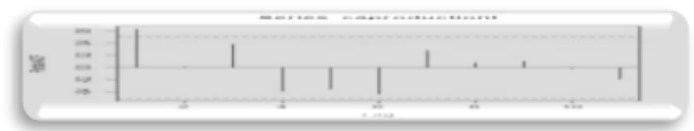

Fig:15 prod-ACF(Coastal Region)

Table-2 Area, Yield And Production ACF And PACF(CEDED REGION \& COASTAL ANDHRA REGIO

\begin{tabular}{|c|c|c|c|c|c|c|c|c|c|c|c|c|}
\hline & \multicolumn{6}{|c|}{ Area, Yield and Production ACF and PACF(CEDED REGION) } & \multicolumn{6}{|c|}{ Area, Yield and Production ACF and PACF(COASTAL REGION) } \\
\hline Lag & $\begin{array}{l}\text { ACF } \\
\text { (area) }\end{array}$ & $\begin{array}{l}\text { PACF } \\
\text { (area) }\end{array}$ & $\begin{array}{l}\text { ACF } \\
\text { (YIELD) }\end{array}$ & $\begin{array}{l}\text { PACF } \\
\text { (YIELD) }\end{array}$ & $\begin{array}{l}\mathrm{ACF} \\
\text { (PROD) }\end{array}$ & $\begin{array}{l}\text { PACF } \\
\text { (PROD) }\end{array}$ & $\begin{array}{l}\mathrm{ACF} \\
\text { (AREA) }\end{array}$ & $\begin{array}{l}\text { PACF } \\
\text { (AREA) }\end{array}$ & $\begin{array}{l}\mathrm{ACF} \\
\text { (YIELD) }\end{array}$ & $\begin{array}{l}\text { PACF } \\
\text { (YIELD) }\end{array}$ & $\begin{array}{l}\mathrm{ACF} \\
(\mathrm{PRO})\end{array}$ & $\begin{array}{l}\text { PACF } \\
\text { (PROD.) }\end{array}$ \\
\hline 0 & 1.000 & 0 & 1.000 & 0 & 1.000 & 0 & 1.000 & 0 & 1.000 & 0 & 1.000 & 0 \\
\hline 1 & 0.299 & 0.299 & -0.306 & -0.306 & -0.348 & -0.348 & 0.784 & 0.784 & 0.546 & 0.546 & 0.634 & 0.634 \\
\hline
\end{tabular}


International Journal of Research in Advent Technology, Vol.7, No.11, November 2019 E-ISSN: 2321-9637

Available online at www.ijrat.org

\begin{tabular}{|c|c|c|c|c|c|c|c|c|c|c|c|c|}
\hline 2 & -0.030 & \begin{tabular}{l|l|}
-0.131 \\
\end{tabular} & 0.119 & 0.028 & $\mathbf{- 0 . 0 3 7}$ & -0.180 & 0.578 & -0.097 & 0.287 & -0.016 & 0.410 & 0.014 \\
\hline 3 & 0.006 & 0.062 & 0.081 & 0.138 & 0.449 & 0.437 & 0.477 & 0.148 & 0.369 & 0.311 & 0.493 & 0.381 \\
\hline 4 & 0.205 & 0.202 & -0.123 & -0.073 & -0.318 & -0.025 & 0.287 & -0.314 & 0.242 & -0.126 & .256 & $\begin{array}{l}-0.390 \\
\end{array}$ \\
\hline 5 & $\begin{array}{l}-0.057 \\
\end{array}$ & -0.217 & $\begin{array}{l}-0.041 \\
\end{array}$ & -0.135 & 0.023 & -0.104 & 0.038 & 0.248 & -0.008 & -0.185 & $\begin{array}{c}-0.103 \\
\end{array}$ & $\begin{array}{l}-0.355 \\
\end{array}$ \\
\hline 6 & $\begin{array}{l}-0.201 \\
\end{array}$ & -0.100 & 0.033 & -0.008 & 0.046 & $\begin{array}{c}-0.240 \\
\end{array}$ & -0.154 & -0.175 & -0.143 & -0.208 & -0.274 & $\begin{array}{l}-0.437 \\
\end{array}$ \\
\hline 7 & 0.016 & 0.140 & -0.152 & -0.115 & -0.204 & $-\mathbf{- 0 . 1 3 7}$ & -0.263 & 0.008 & -0.120 & 0.007 & -0.228 & 0.280 \\
\hline 8 & -0.051 & -0.221 & 0.115 & 0.046 & 0.027 & -0.054 & -0.368 & -0.077 & -0.114 & 0.045 & -0.331 & 0.078 \\
\hline 9 & $\begin{array}{c}-0.130 \\
\end{array}$ & -0.002 & -0.228 & -0.206 & -0.161 & -0.191 & -0.450 & 0.038 & -0.225 & -0.077 & $\begin{array}{c}-0.480 \\
\end{array}$ & 0.108 \\
\hline 10 & -0.209 & -0.104 & 0.284 & 0.208 & 0.054 & $\mathbf{0 . 0 4 7}$ & -0.432 & 0.045 & -0.396 & -0.316 & -0.311 & -0.014 \\
\hline 11 & $-\mathbf{- 0 . 1 0 8}$ & -0.145 & -0.077 & 0.061 & $-\mathbf{- 0 . 0 3 3}$ & -0.014 & -0.340 & 0.099 & -0.275 & 0.048 & -0.189 & -0.187 \\
\hline 12 & $-\mathbf{- 0 . 1 3 0}$ & -0.048 & -0.112 & -0.181 & 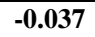 & 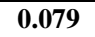 & -0.274 & -0.044 & -0.146 & 0.042 & \begin{tabular}{c|}
-0.208 \\
\end{tabular} & $\begin{array}{l}-0.104 \\
\end{array}$ \\
\hline 13 & 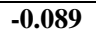 & 0.001 & 0.010 & -0.185 & 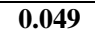 & -0.118 & -0.250 & -0.185 & -0.288 & -0.107 & 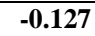 & -0.066 \\
\hline 14 & $\begin{array}{c}-0.020 \\
\end{array}$ & $\begin{array}{l}-0.030 \\
\end{array}$ & $\begin{array}{l}-0.094 \\
\end{array}$ & -0.113 & $\begin{array}{c}-0.010 \\
\end{array}$ & $\begin{array}{c}-0.060 \\
\end{array}$ & -0.134 & 0.068 & -0.228 & 0.067 & -0.042 & -0.145 \\
\hline
\end{tabular}

Table-3 AREA, YIELD, AND PRODUCTTION POINT FORECAST (CEDED REGION)

\begin{tabular}{|c|c|c|c|c|c|c|}
\hline \multicolumn{6}{|c|}{ Area Point Forecast of Groundnut (CEDED REGION) } & \multirow{3}{*}{$\begin{array}{l}\text { Fig: } 16 \text { Area forecast } \\
\text { rerecasto from aremea(1,2,1) }\end{array}$} \\
\hline Year & $\begin{array}{l}\text { Area Point } \\
\text { forecast }\end{array}$ & Lo 80 & Hi 80 & Lo95 & Hi 95 & \\
\hline 2018 & 663.6431 & 74.34483 & 1252.941 & -237.6108 & 1564.897 & \\
\hline 2019 & 630.2862 & -230.43900 & 1491.011 & \multirow{2}{*}{$\begin{array}{l}-686.0794 \\
-1064.9008\end{array}$} & 1946.652 & \\
\hline 2020 & 596.9294 & -489.68298 & 1683.542 & & 2258.760 & \\
\hline 2021 & $\mathbf{5 6 3 . 5 7 2 5}$ & -727.51535 & 1854.660 & -1410.9760 & 2538.121 & \\
\hline 2022 & 530.2156 & -952.81919 & 2013.250 & -1737.8904 & 2798.322 & \\
\hline 2023 & $\begin{array}{l}96.8587 \\
\end{array}$ & -1169.92849 & 2163.646 & -2052.2723 & 3045.990 & \\
\hline 2024 & 463.5018 & -1381.29353 & 2308.297 & -2357.8692 & 3284.873 & \\
\hline 2025 & 430.1449 & -1588.43457 & 2448.724 & -2657.0060 & 3517.296 & sois \\
\hline 2026 & 396.7881 & -1792.35779 & 2585.934 & -2951.2216 & 3744.798 & \\
\hline 2027 & 363.4312 & -1993.76182 & 2720.624 & -3241.5844 & 3968.447 & \\
\hline \multicolumn{6}{|c|}{ Yield Point Forecast (CEDED REGION) } & Fig:17 Yield forecast \\
\hline Year & $\begin{array}{l}\text { Yield point } \\
\text { forecast }\end{array}$ & Lo 80 & Hi 80 & Lo95 & Hi 95 & \\
\hline 2018 & 4338.339 & 2588.741 & 6087.937 & 1662.5598 & 1662.5598 & \\
\hline 2019 & 5078.286 & 3321.278 & 6835.294 & 2391.1738 & 7765.398 & \\
\hline 2020 & 5563.951 & 3536.040 & 7591.862 & 2462.5287 & 8665.374 & \\
\hline 2021 & \begin{tabular}{|c|}
5098.539 \\
\end{tabular} & 2814.759 & 7382.319 & 1605.7985 & 8591.279 & \\
\hline 2022 & 5657.126 & 3256.581 & \begin{tabular}{|l|}
8057.670 \\
\end{tabular} & \multirow{2}{*}{\begin{tabular}{|l|}
1985.8098 \\
16118634
\end{tabular}} & 9328.442 & \\
\hline 2023 & 5666.277 & 3015.238 & 8317.315 & & 9720.690 & \\
\hline 2024 & $\begin{array}{l}5738.279 \\
\end{array}$ & 2906.801 & 8569.757 & \multirow{2}{*}{\begin{tabular}{|l|}
1407.9071 \\
1386.2738 \\
\end{tabular}} & 10068.650 & \\
\hline 2025 & 5998.293 & 2982.656 & 9013.931 & & 10610.313 & $=$ \\
\hline 2026 & 6050.198 & 2823.687 & 9276.709 & 1115.6751 & 10984.720 & \\
\hline 2027 & 6216.148 & 2804.532 & 9627.765 & 998.5315 & 11433.765 & \\
\hline Produc & on Point Forec & cast (CEDED RF & QGION) & & & Fig:18 Production forecast \\
\hline Year & $\begin{array}{l}\text { Production } \\
\text { Point forecas }\end{array}$ & Lo 80 & Hi 80 & $\mathbf{L 0 9 5}$ & Hi 95 & \\
\hline 2018 & 670.0918 & -116.26186 & 1456.446 & -532.5324 & 1872.716 & \\
\hline 2019 & 818.4277 & -56.75061 & 1693.606 & -520.0420 & 2156.898 & \\
\hline 2020 & 724.4765 & -376.64553 & 1825.599 & -959.5443 & 2408.497 & \\
\hline 2021 & 770.2133 & -461.00217 & 2001.429 & -1112.7683 & 2653.195 & \\
\hline 2022 & 735.4144 & -663.80745 & 2134.636 & -1404.5108 & 2875.340 & \\
\hline 2023 & 747.0475 & -792.33296 & 2286.428 & -1607.2318 & 3101.327 & \\
\hline 2024 & 731.9108 & -957.86174 & 2421.683 & -1852.3733 & 3316.195 & \\
\hline 2025 & 732.2079 & 1098.65717 & \begin{tabular}{|l|}
2563.073 \\
\end{tabular} & -2067.8586 & 3532.274 & \\
\hline 2026 & 723.6068 & -1250.72629 & 2697.940 & -2295.8751 & 3743.089 & \\
\hline 2027 & 720.1359 & 1394.13130 & \begin{tabular}{|l|}
2834.403 \\
\end{tabular} & -2513.3568 & 3953.629 & $=$ \\
\hline
\end{tabular}


International Journal of Research in Advent Technology, Vol.7, No.11, November 2019

E-ISSN: 2321-9637

Available online at www.ijrat.org

Table -4 AREA, YIELD, AND PRODUCTTION POINT FORECAST(COASTAL ANDHRA REGION)

\begin{tabular}{|c|c|c|c|c|c|}
\hline \multicolumn{6}{|c|}{ Area Point Forecast of Groundnut (COASTAL REGION) } \\
\hline Year & $\begin{array}{l}\text { Area Point } \\
\text { forecast }\end{array}$ & Lo 80 & Hi 80 & Lo95 & Hi 95 \\
\hline 2018 & 30.313146 & 13.4849095 & 47.14138 & 47.14138 & 56.04971 \\
\hline 2019 & 23.270686 & 0.4993657 & 46.04201 & -11.555043 & 58.09642 \\
\hline 2020 & 16.282895 & -11.9052322 & 44.47102 & 44.47102 & 59.39292 \\
\hline 2021 & 9.286700 & -23.8423228 & 42.41572 & -41.379768 & 59.95317 \\
\hline 2022 & 2.291796 & -35.5194736 & 40.10307 & -55.535551 & 60.11914 \\
\hline 2023 & -4.703306 & -47.0197310 & 37.61312 & -69.420693 & 60.01408 \\
\hline 2024 & -11.698378 & -58.3947964 & 34.99804 & -83.114387 & 59.71763 \\
\hline 2025 & -18.693454 & -69.6768774 & 32.28997 & -96.665871 & 59.27896 \\
\hline 2026 & -25.688529 & -80.8876528 & 29.51059 & -110.108304 & 58.73124 \\
\hline 2027 & -32.683605 & -92.0423157 & 26.67511 & -123.464919 & 58.09771 \\
\hline
\end{tabular}

Yield Point Forecast(COASTAL REGION)

\begin{tabular}{|l|l|l|l|l|l|}
\hline Year & $\begin{array}{l}\text { Yield point } \\
\text { forecast }\end{array}$ & Lo 80 & Hi 80 & Lo95 & Hi 95 \\
\hline $\mathbf{2 0 1 8}$ & 25404.50 & 22497.42 & 28311.58 & 20958.51 & 29850.49 \\
\hline $\mathbf{2 0 1 9}$ & 26290.00 & 22043.95 & 30536.06 & 19796.22 & 32783.79 \\
\hline $\mathbf{2 0 2 0}$ & 27175.51 & 21815.12 & 32535.89 & 18977.50 & 35373.51 \\
\hline $\mathbf{2 0 2 1}$ & 28061.01 & 21691.92 & 34430.10 & 18320.33 & 37801.69 \\
\hline $\mathbf{2 0 2 2}$ & 28946.51 & 21630.53 & 36262.50 & 17757.68 & 40135.35 \\
\hline $\mathbf{2 0 2 3}$ & 29832.01 & 21609.56 & 38054.47 & 17256.85 & 42407.18 \\
\hline $\mathbf{2 0 2 4}$ & 30717.52 & 21616.92 & 39818.11 & 16799.36 & 44635.67 \\
\hline $\mathbf{2 0 2 5}$ & 31603.02 & 21645.13 & 41560.91 & 16373.74 & 46832.30 \\
\hline $\mathbf{2 0 2 6}$ & 32488.52 & 21689.21 & 43287.84 & 15972.39 & 49004.65 \\
\hline $\mathbf{2 0 2 7}$ & 33374.02 & 21745.71 & 45002.33 & 15590.06 & 51157.99 \\
\hline
\end{tabular}

Fig:20 Yield forecast

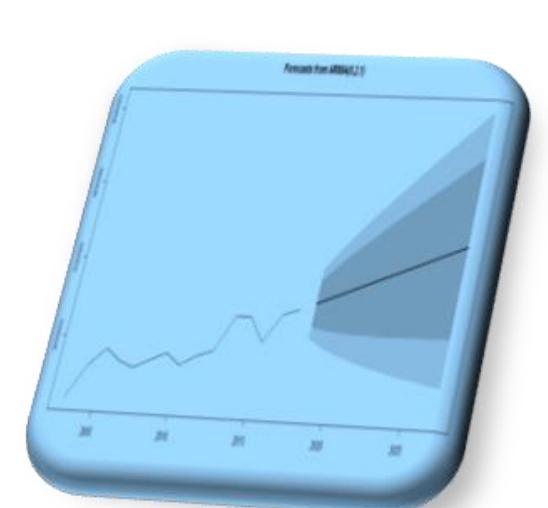

Production Point Forecast(COASTAL REGION)

\begin{tabular}{|l|l|l|l|l|l|}
\hline Year & $\begin{array}{l}\text { Production } \\
\text { Point forecas }\end{array}$ & Lo 80 & Hi 80 & Lo95 & Hi 95 \\
\hline $\mathbf{2 0 1 8}$ & 129.4848 & 105.739621 & 153.2300 & 93.16968 & 165.7999 \\
\hline $\mathbf{2 0 1 9}$ & 122.6794 & 90.200070 & 155.1586 & 73.00657 & 172.3521 \\
\hline $\mathbf{2 0 2 0}$ & 108.9265 & 71.588542 & 146.2645 & 51.82301 & 166.0300 \\
\hline $\mathbf{2 0 2 1}$ & 120.9781 & 70.405265 & 171.5510 & 43.63361 & 198.3226 \\
\hline $\mathbf{2 0 2 2}$ & 122.8605 & 58.419666 & 187.3014 & 24.30673 & 221.4143 \\
\hline $\mathbf{2 0 2 3}$ & 112.2065 & 37.588853 & 186.8241 & -1.91134 & 226.3243 \\
\hline $\mathbf{2 0 2 4}$ & 116.2073 & 27.936700 & 204.4780 & -18.79096 & 251.2057 \\
\hline $\mathbf{2 0 2 5}$ & 120.5945 & 16.284411 & 224.9046 & -38.93402 & 280.1230 \\
\hline $\mathbf{2 0 2 6}$ & 114.2439 & -4.218647 & 232.7065 & -66.92895 & 295.4168 \\
\hline $\mathbf{2 0 2 7}$ & 113.9214 & -19.846723 & 247.6895 & -90.65927 & 318.5020 \\
\hline
\end{tabular}

Fig:21 Production forecast
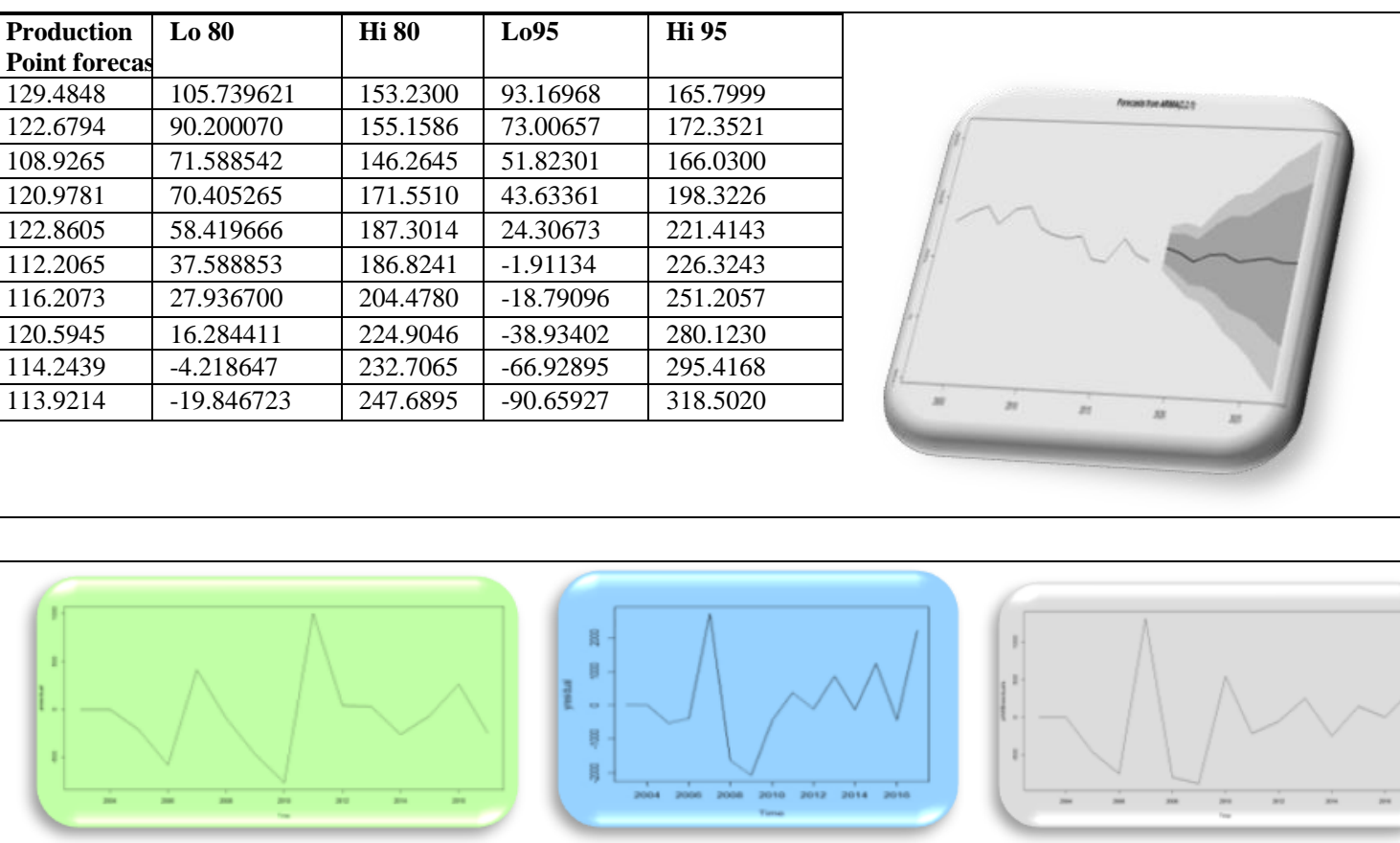

Fig:22. Area-Residuals(CEDED)

Fig:23:Yield-Residua(Ceded)

Fig:24 Prod.-Residuals(Ceded) 
International Journal of Research in Advent Technology, Vol.7, No.11, November 2019 E-ISSN: 2321-9637

Available online at www.ijrat.org

Table-5 Residuals \& Predictive values of Area, Yield and Productions(CEDED REGION)

\begin{tabular}{|c|c|c|c|c|c|c|}
\hline year & A residuals & Y residuals & P residuals & A predective & Y predictive & P predective \\
\hline 2003 & 0.5205565 & 1.191376 & 0.2696697 & 1164.5206 & 266.1914 & 603.2697 \\
\hline 2004 & -0.7857512 & -1.805380 & 0.6757360 & 1510.2142 & 3453.1946 & 1267.6757 \\
\hline 2005 & -214.9619841 & -533.095212 & -463.3631946 & 1339.0380 & 2266.9048 & 460.6368 \\
\hline 2006 & -578.0808788 & -397.622113 & -754.3694676 & 462.9191 & 2124.3779 & -388.3695 \\
\hline 2007 & 410.4950817 & 2709.383368 & 1316.2430014 & 1884.4951 & 8987.3834 & 3392.2430 \\
\hline 2008 & -93.4683524 & -1638.368125 & -797.0453050 & 1353.5316 & 339.6319 & -326.0453 \\
\hline 2009 & -467.9382883 & -2070.179756 & -883.6285771 & 523.0617 & 517.8202 & -390.6286 \\
\hline 2010 & -764.8823535 & -450.995919 & 540.5922361 & -628.8824 & 3015.0041 & 1502.5922 \\
\hline 2011 & 999.8238396 & 370.213894 & -217.0168277 & 2057.8238 & 3051.2139 & 226.9832 \\
\hline 2012 & 41.7188844 & -116.687055 & -57.7366664 & 1130.7189 & 2783.3129 & 577.2633 \\
\hline 2013 & 28.7763566 & 859.505100 & 252.7839521 & 1139.7764 & 4792.5051 & 991.7840 \\
\hline 2014 & -260.9630661 & -144.666116 & -250.2469229 & 571.0369 & 2629.3339 & 140.7531 \\
\hline 2015 & -66.8461418 & 1241.299335 & 145.7239208 & 665.1539 & 6026.2993 & 839.7239 \\
\hline 2016 & 261.3288712 & -442.772159 & 2.3333701 & 1229.3289 & 2669.2278 & 487.3334 \\
\hline 2017 & -246.6139287 & 2196.844463 & 358.7155252 & 450.3861 & 8439.8445 & 1300.7155 \\
\hline
\end{tabular}

Table-6 Residuals \& Predictive values of Area, Yield and Productions(COASTAL ANDHRA REGION)

\begin{tabular}{|c|c|c|c|c|c|c|}
\hline year & A residuals & Y residuals & $P$ residuals & A predective & $\mathbf{Y}$ predictive & $P$ redective \\
\hline 2003 & 0.05992661 & 5.421122 & 0.07110694 & 134.05993 & 12127.42 & 159.07111 \\
\hline 2004 & -0.17530698 & -7.035089 & -0.17754246 & 135.82469 & 16241.96 & 174.82246 \\
\hline 2005 & -9.75765955 & 1334.323530 & -2.62360133 & 113.24234 & 17154.68 & 183.37640 \\
\hline 2006 & -3.70558785 & -3766.917577 & -24.31993545 & 110.29441 & 13292.08 & 132.68006 \\
\hline 2007 & 6.55777815 & -2150.927389 & 19.73043580 & 121.55778 & 14070.07 & 202.73044 \\
\hline 2008 & 8.12492792 & 39.571429 & -8.45449021 & 126.12493 & 17329.57 & 179.54551 \\
\hline 2009 & -15.87964846 & -6.943517 & -15.82348111 & 80.12035 & 18309.06 & 138.17652 \\
\hline 2010 & -1.17360226 & -2309.309307 & \begin{tabular}{|c|}
-22.50726628 \\
\end{tabular} & 89.82640 & 14544.69 & 120.49273 \\
\hline 2011 & -6.96986748 & 531.311061 & -19.24844333 & 70.03013 & 18629.31 & 118.75156 \\
\hline 2012 & -0.83489672 & -48.087117 & 20.78926920 & 69.16510 & 18745.91 & 163.78927 \\
\hline 2013 & -19.61687161 & 3508.860583 & -21.85512723 & 22.38313 & 26742.86 & 84.14487 \\
\hline 2014 & 5.89437125 & -1017.538421 & 5.02948856 & 47.89437 & 22260.46 & 107.02949 \\
\hline 2015 & 32.44007125 & -3902.650475 & 35.66558397 & 98.44007 & 16313.35 & 177.66558 \\
\hline 2016 & -8.91152621 & 2760.767403 & 1.60879507 & 37.08847 & 26524.77 & 118.60880 \\
\hline 2017 & -4.15845853 & -135.428640 & 8.16232185 & 32.84154 & 24383.57 & 114.16232 \\
\hline \multicolumn{6}{|c|}{ Fig:26:Yield-Residuals } & Fig:27 Prod.- $\quad$ Residuals \\
\hline
\end{tabular}

Table-7 Area, yield , and Production predictive (CEDED REGION \& Coastal Region)

\begin{tabular}{|l|l|l|l|l|l|l|}
\hline & \multicolumn{5}{|l|}{ CEDED REGION } & \multicolumn{2}{l|}{ Coastal Region } \\
\hline year & $\begin{array}{l}\text { Area } \\
\text { predictive }\end{array}$ & $\begin{array}{l}\text { Yield } \\
\text { predictive }\end{array}$ & $\begin{array}{l}\text { Prod. } \\
\text { predective }\end{array}$ & $\begin{array}{l}\text { A REA } \\
\text { Predictive }\end{array}$ & $\begin{array}{l}\text { YIELD } \\
\text { predictive }\end{array}$ & $\begin{array}{l}\text { P RODUCTION } \\
\text { predictive }\end{array}$ \\
\hline $\mathbf{2 0 1 8}$ & $\mathbf{6 6 3 . 6 4 3 1 2}$ & $\mathbf{4 3 3 8 . 3 3 9}$ & $\mathbf{6 7 0 . 0 9 1 8}$ & $\mathbf{3 0 . 3 1 3 1 4 6}$ & $\mathbf{2 5 4 0 4 . 5 0}$ & $\mathbf{1 8 . 5 2 8 4 7}$ \\
\hline 2019 & $\mathbf{6 3 0 . 2 8 6 2 4}$ & $\mathbf{5 0 7 8 . 2 8 6}$ & $\mathbf{8 1 8 . 4 2 7 7}$ & $\mathbf{2 3 . 2 7 0 6 8 6}$ & $\mathbf{2 6 2 9 0 . 0 0}$ & $\mathbf{2 5 . 3 4 3 7 2}$ \\
\hline $\mathbf{2 0 2 0}$ & $\mathbf{5 9 6 . 9 2 9 3 6}$ & $\mathbf{5 5 6 3 . 9 5 1}$ & $\mathbf{7 2 4 . 4 7 6 5}$ & 16.282895 & $\mathbf{2 7 1 7 5 . 5 1}$ & $\mathbf{2 9 . 1 3 4 9 8}$ \\
\hline $\mathbf{2 0 2 1}$ & $\mathbf{5 6 3 . 5 7 2 4 7}$ & $\mathbf{5 0 9 8 . 5 3 9}$ & $\mathbf{7 7 0 . 2 1 3 3}$ & $\mathbf{9 . 2 8 6 7 0 0}$ & $\mathbf{2 8 0 6 1 . 0 1}$ & $\mathbf{3 9 . 4 6 2 2 1}$ \\
\hline $\mathbf{2 0 2 2}$ & $\mathbf{5 3 0 . 2 1 5 5 9}$ & $\mathbf{5 6 5 7 . 1 2 6}$ & $\mathbf{7 3 5 . 4 1 4 4}$ & $\mathbf{2 . 2 9 1 7 9 6}$ & $\mathbf{2 8 9 4 6 . 5 1}$ & $\mathbf{5 0 . 2 8 3 4 7}$ \\
\hline $\mathbf{2 0 2 3}$ & $\mathbf{4 9 6 . 8 5 8 7 1}$ & $\mathbf{5 6 6 6 . 2 7 7}$ & $\mathbf{7 4 7 . 0 4 7 5}$ & $\mathbf{- 4 . 7 0 3 3 0 6}$ & $\mathbf{2 9 8 3 2 . 0 1}$ & $\mathbf{5 8 . 2 2 4 4 6}$ \\
\hline $\mathbf{2 0 2 4}$ & $\mathbf{4 6 3 . 5 0 1 8 3}$ & $\mathbf{5 7 3 8 . 2 7 9}$ & $\mathbf{7 3 1 . 9 1 0 8}$ & $\mathbf{- 1 1 . 6 9 8 3 7 8}$ & $\mathbf{3 0 7 1 7 . 5 2}$ & $\mathbf{6 8 . 8 7 7 9 5}$ \\
\hline
\end{tabular}


International Journal of Research in Advent Technology, Vol.7, No.11, November 2019 E-ISSN: 2321-9637

Available online at www.ijrat.org

\begin{tabular}{|c|c|c|c|c|c|c|}
\hline 2025 & 430.14495 & 5998.293 & 732.2079 & -18.693454 & 31603.02 & 81.39360 \\
\hline 2026 & 396.78807 & 6050.198 & 723.6068 & -25.68529 & 32488.52 & 92.43685 \\
\hline 2027 & 363.43118 & 6216.148 & 720.1359 & -32.683605 & 33374.02 & 104.37980 \\
\hline 2028 & 330.07430 & 6367.048 & 713.7073 & -39.678681 & 34259.53 & 118.01384 \\
\hline 2029 & 296.71742 & 6480.838 & 708.9839 & -46.673756 & 35145.03 & 131.27342 \\
\hline 2030 & 263.36054 & 6636.899 & 703.2773 & -53.668832 & 36030.53 & 144.74957 \\
\hline 2031 & 230.00366 & 6769.308 & 698.1376 & -60.663908 & 36916.03 & 159.43533 \\
\hline 2032 & 196.64678 & 6905.240 & 692.6710 & -67.658983 & 37801.53 & 174.34269 \\
\hline 2033 & 163.28990 & 7048.485 & 687.3929 & -74.654059 & 38687.04 & 189.32485 \\
\hline 2034 & 129.93301 & 7183.148 & 682.0062 & -81.649135 & 39572.54 & 205.09936 \\
\hline 2035 & 96.57613 & 7322.712 & 676.6820 & -88.644210 & 40458.04 & 221.33180 \\
\hline 2036 & 63.21925 & 7461.466 & 671.3218 & -95.639286 & 41343.54 & 237.69702 \\
\hline 2037 & 29.86237 & 7598.782 & 665.9824 & -102.634362 & 42229.05 & 254.58425 \\
\hline
\end{tabular}

Table-8 Time series data values of Area, Yield and Production(CEDED REGION \& COASTAL REGION) CEDED REGION

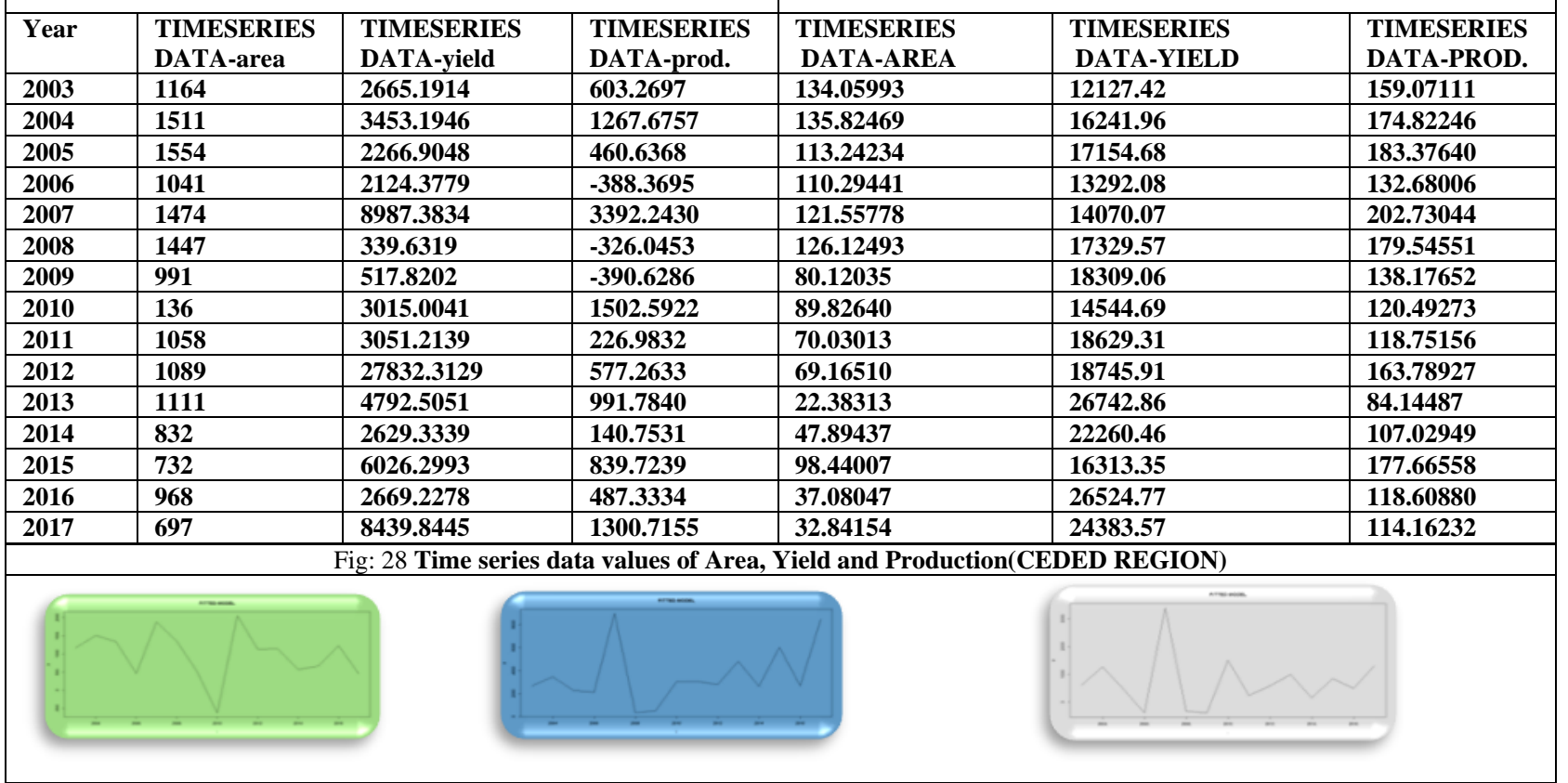

Fig: 29 Time series data values of Area, Yield and Production(COASTAL REGION)
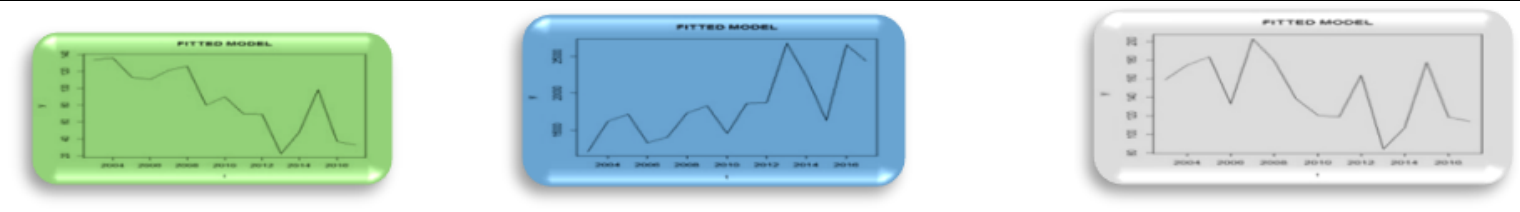

Table-9 Area, yield and Production Training Set error measure

\begin{tabular}{|c|c|c|c|c|c|c|c|c|}
\hline \multirow[b]{2}{*}{ CEDED REGION } & \multirow[b]{2}{*}{ ARIMA } & \multicolumn{7}{|c|}{ Training set error measures } \\
\hline & & ME & RMSE & MAE & MPE & MAPE & MASE & ACF \\
\hline AREA & $(1,2,1)$ & -63.45848 & 413.5649 & 295.8136 & -40.37166 & 61.14672 & 0.913206 & -0.2363987 \\
\hline YIELD & $(0,2,1)$ & 105.483 & 1222.83 & 878.3086 & -60277613 & 24.9433 & 0.5778618 & -0.13744871 \\
\hline PRODUCTION & $(2,1,2)$ & -53.73797 & 550.8458 & 402.7163 & $-\mathbf{3 4 . 2 1 5 3}$ & 62.67673 & 0.7516369 & -0.388102 \\
\hline \multicolumn{9}{|l|}{$\begin{array}{l}\text { COASTAL } \\
\text { ANDHRA REGION }\end{array}$} \\
\hline AREA & $(\mathbf{1}, 2,1)$ & -1.20709 & 11.80239 & 8.284033 & -2.725881 & 12.83502 & 0.7387036 & -0.1005916 \\
\hline YIELD & $(0,2,1)$ & -522.2153 & 2040.165 & 1435.006 & -3.393128 & 7.383666 & 0.7732607 & -0.05819921 \\
\hline PRODUCTION & $(1,2,1)$ & -1.596859 & $\mathbf{1 7 . 2 4 9 1 4}$ & 13.73779 & -1.1728569. & 9.771143 & 0.7425834 & -0.09666837 \\
\hline
\end{tabular}


International Journal of Research in Advent Technology, Vol.7, No.11, November 2019

E-ISSN: 2321-9637

Available online at www.ijrat.org

V. Conclusion

A. Area of Groundnut Crop Conclusion

Table-10 Identification of ARIMA(p,d,q) MODEL for AREA in Ceded \& Coastal Andhra Regions

\begin{tabular}{|c|c|c|c|c|c|c|c|}
\hline \multicolumn{8}{|c|}{ Identification of ARIMA(p,d,q) MODEL for AREA (Ceded Region) } \\
\hline Model & $\begin{array}{l}\text { Area } \\
\text { ARIMA }\end{array}$ & Coefficients & SE & Intercept & $\sigma^{\wedge} \mathbf{2}$ & $\begin{array}{l}\text { Log } \\
\text { likelyhood }\end{array}$ & AIC \\
\hline \multirow[t]{2}{*}{$(\mathbf{1 , 0 , 1 )}$} & AR1 & 0.0498 & 0.5624 & 1046.008 & \multirow[t]{2}{*}{115069} & \multirow[t]{2}{*}{-108.74} & \multirow[t]{2}{*}{225.49} \\
\hline & MA1 & 0.2924 & 0.5138 & $\mathbf{1 1 7 . 2 3 3}$ & & & \\
\hline \multirow[t]{4}{*}{$(1,1,1)$} & AR1 & 0.1970 & 0.3672 & & \multirow[t]{4}{*}{134509} & \multirow[t]{4}{*}{-102.82} & \multirow[t]{4}{*}{211.65} \\
\hline & MA1 & -0.7633 & 0.2536 & & & & \\
\hline & AR2 & -0.3039 & 0.2835 & & & & \\
\hline & MA1 & -0.5565 & 0.3623 & & & & \\
\hline$(0,1,1)$ & MA1 & $\begin{array}{l}-0.6696 \\
\end{array}$ & 0.2105 & & 137404 & $\begin{array}{l}-102.98 \\
\end{array}$ & 209.95 \\
\hline \multirow[t]{2}{*}{$(1,2,1)$} & AR1 & -0.2117 & 0.2744 & & \multirow[t]{2}{*}{183257} & \multirow[t]{2}{*}{-98.74} & \multirow[t]{2}{*}{203.48} \\
\hline & MA1 & -1.000 & 0.2106 & & & & \\
\hline$(1,1,0)$ & AR1 & -0.2581 & 0.2570 & & 171256 & -104.26 & 212.51 \\
\hline \multirow[t]{3}{*}{$(1,1,2)$} & AR1 & $\begin{array}{l}-0.1588 \\
\end{array}$ & 0.7451 & & \multirow[t]{3}{*}{131574} & \multirow[t]{3}{*}{-102.68} & \multirow[t]{3}{*}{213.35} \\
\hline & MA1 & -0.3572 & 0.6960 & & & & \\
\hline & MA2 & -0.2946 & 0.4351 & & & & \\
\hline$(1,2,0)$ & AR1 & $\begin{array}{l}-0.4470 \\
\end{array}$ & 0.2389 & & 378348 & $\begin{array}{l}-102.04 \\
\end{array}$ & 208.08 \\
\hline$(0,2,1)$ & MA1 & -1.000 & 0.2025 & & 197349 & -99.02 & 202.04 \\
\hline \multicolumn{8}{|c|}{ Identification of ARIMA(p,d,q) MODEL for AREA in (Coastal Andhra Region) } \\
\hline model & $\begin{array}{l}\text { Area } \\
\text { ARIMA }\end{array}$ & Coefficients & SE & Intercept & $\sigma^{\wedge} 2$ & $\begin{array}{l}\text { Log } \\
\text { likelyhood }\end{array}$ & AIC \\
\hline \multirow[t]{2}{*}{$(\mathbf{1}, \mathbf{0 , 1})$} & AR1 & 0.9190 & 0.1088 & 85.7207 & \multirow[t]{2}{*}{198.3} & \multirow[t]{2}{*}{-62.03} & \multirow[t]{2}{*}{132.07} \\
\hline & MA1 & 0.1533 & 0.3450 & $\begin{array}{l}32.5630 \\
\end{array}$ & & & \\
\hline \multirow[t]{2}{*}{$\begin{array}{l}(1,1,1) \\
\end{array}$} & AR1 & $\begin{array}{l}-0.8244 \\
\end{array}$ & 0.2173 & & \multirow[t]{2}{*}{184.7} & \multirow[t]{2}{*}{-56.82} & \multirow[t]{2}{*}{119.65} \\
\hline & MA1 & 0.9999 & 0.4058 & & & & \\
\hline \multirow[t]{3}{*}{$(2,2,1)$} & AR1 & -0.2573 & 0.2572 & & \multirow[t]{3}{*}{131.5} & -52.07 & 112.14 \\
\hline & AR2 & -0.3591 & 0.2522 & & & & \\
\hline & MA1 & -1.0000 & 0.2971 & & & & \\
\hline$(1,2,2)$ & AR1 & 0.4313 & 0.3430 & & 114.8 & -52.14 & 112.29 \\
\hline & MA1 & -1.9696 & 0.4247 & & & & \\
\hline & MA2 & 0.9999 & 0.4251 & & & & \\
\hline$(1,1,0)$ & AR1 & 0.0648 & 0.2612 & & 203.3 & -57.07 & 118.14 \\
\hline$(\mathbf{0 , 0 , 1 )}$ & MA1 & 0.9157 & 0.2666 & 86.4312 & 431 & -67.66 & 141.32 \\
\hline & & & & 10.0006 & & & \\
\hline$(1,2,0)$ & AR1 & -0.4331 & 0.2426 & & 321.5 & -56.07 & 116.15 \\
\hline$(\mathbf{1 , 0 , 0 )}$ & AR1 & 0.9418 & 0.702 & 86.0175 & 200.7 & -62.14 & 130.27 \\
\hline & & & & 35.3574 & & & \\
\hline
\end{tabular}

In the present study, the ARIMA $(1,2,1)$ in Ceded Region \& ARIMA $(2,2,1)$ in Coastal Region were the best fitted model through the minimum value of AIC, then used for prediction up to 10 years of the area of groundnut in ceded districts using 15 years time series data i.e. from 2003-2004 to2017-2018. ARIMA(1,2,1) in Ceded Region \& ARIMA $(2,2,1)$ in Coastal Region were used because the reason of its capability to make prediction using time series data with any kind of patterns and with auto correlated successive values of the time series. The study was also validated and statistically tested that the successive residuals in the fitted ARIMA (1, 2,1 ) in Ceded Region \& ARIMA $(2,2,1)$ in Coastal Region were not correlated, and the residuals appear to be normally

it can be a satisfactory predictive model for the groundnut area in ceded districts in Andhra Pradesh for the period of 2018 to 2027. The ARIMA $(1,2,1)$ in Ceded Region \& ARIMA $(2,2,1)$ in Coastal Region models projected an increment in the area for the duration of 2018 to 2027 . The prediction of 2027 is resulted approximately 363.4312'000 ha \& $\mathbf{- 3 2 . 6 8 3 6 0 5}$ '000 ha. Like any other predictive models for forecasting, ARIMA model has also limitations on accuracy of the predictions yet it is widely used for forecasting the future values for time series.

\section{B. Yield of Groundnut Crop Conclusion} distributed with the mean zero and constant variance. Hence, Table 11 Identification of ARIMA(p,d,q) MODEL for Yield in Ceded \& Coastal Andhra Regions

\begin{tabular}{|l|l|l|l|l|l|l|l|}
\hline \multicolumn{2}{|c|}{ Identification of ARIMA(p,d,q) } & MODEL for YIELD (CEDED REGION) \\
\hline Model & $\begin{array}{l}\text { YIELD } \\
\text { ARIMA }\end{array}$ & $\begin{array}{l}\text { Coefficie } \\
\text { nts }\end{array}$ & SE & Intercept & $\sigma^{\wedge} 2$ & $\begin{array}{l}\text { loglikely- } \\
\text { hood }\end{array}$ & AIC \\
\hline
\end{tabular}


International Journal of Research in Advent Technology, Vol.7, No.11, November 2019

E-ISSN: 2321-9637

Available online at www.ijrat.org

\begin{tabular}{|c|c|c|c|c|c|c|c|}
\hline \multirow[t]{3}{*}{$(1,2,2)$} & AR1 & -0.4498 & 0.3036 & & \multirow[t]{3}{*}{1951664} & \multirow[t]{3}{*}{-114.66} & \multirow[t]{3}{*}{237.32} \\
\hline & MA1 & -1.4436 & 0.3670 & & & & \\
\hline & MA2 & 0.5442 & 0.3058 & & & & \\
\hline \multirow[t]{3}{*}{$(2,2,1)$} & $\mathbf{A R}$ & -0.9559 & 0.2468 & & \multirow[t]{3}{*}{1725361} & \multirow[t]{3}{*}{-114.44} & \multirow[t]{3}{*}{236.89} \\
\hline & AR2 & $\begin{array}{l}-0.4515 \\
\end{array}$ & 0.2410 & & & & \\
\hline & MA1 & -1.0000 & .7099 & & & & \\
\hline \multirow[t]{3}{*}{$(1,1,2)$} & AR1 & -0.3016 & 0.5638 & & \multirow[t]{3}{*}{1648515} & \multirow[t]{3}{*}{-120.7} & \multirow[t]{3}{*}{249.41} \\
\hline & MA1 & -0.8152 & 0.4808 & & & & \\
\hline & MA2 & 0.2104 & 0.6083 & & & & \\
\hline \multirow[t]{2}{*}{$(1,0,0)$} & \multirow[t]{2}{*}{ AR1 } & \multirow[t]{2}{*}{-0.4288} & \multirow[t]{2}{*}{0.2773} & 3437.8939 & \multirow[t]{2}{*}{1374004} & \multirow[t]{2}{*}{-127.38} & \multirow[t]{2}{*}{260.77} \\
\hline & & & & 217.0428 & & & \\
\hline \multirow[t]{2}{*}{$(\mathbf{0 , 0 , 1 )}$} & \multirow[t]{2}{*}{ MA1 } & \multirow[t]{2}{*}{-0.4099} & \multirow[t]{2}{*}{0.3307} & 3423.1423 & \multirow[t]{2}{*}{1403675} & \multirow[t]{2}{*}{-127.54} & \multirow[t]{2}{*}{261.07} \\
\hline & & & & 195.9421 & & & \\
\hline \multirow{2}{*}{$(\mathbf{1 , 0 , 1 )}$} & AR1 & $\begin{array}{l}-0.4054 \\
\end{array}$ & 0.5620 & 3437.076 & \multirow[t]{2}{*}{1373603} & \multirow[t]{2}{*}{-127.38} & \multirow[t]{2}{*}{262.77} \\
\hline & MA1 & -0.0303 & 0.6045 & 215.595 & & & \\
\hline \multicolumn{8}{|c|}{ Identification of ARIMA(p,d,q) MODEL for YIELD(COASTAL ANDHRA REGION) } \\
\hline Model & $\begin{array}{l}\text { YIELD } \\
\text { ARIMA }\end{array}$ & Coefficients & SE & Intercept & $\sigma^{\wedge} \mathbf{2}$ & $\begin{array}{l}\text { loglikely- } \\
\text { hood }\end{array}$ & AIC \\
\hline \multirow[t]{2}{*}{$(1,1,1)$} & AR1 & 0.3432 & 0.7610 & & 5185326 & -128.1 & 262.21 \\
\hline & MA1 & -0.4558 & 0.6856 & & & & \\
\hline$(2,1,1)$ & AR1 & -0.3786 & 1.0185 & & 4269286 & -127.02 & 262.03 \\
\hline & AR2 & -0.4795 & 0.3230 & & & & \\
\hline & MA1 & 0.3476 & 1.2693 & & & & \\
\hline$(1,0,1)$ & AR1 & 0.4863 & 0.4462 & 18661.461 & 4814922 & -137.4 & 282.8 \\
\hline & MA1 & 0.667 & 00.478 & 1712.654 & & & \\
\hline$(0,2,1)$ & MA1 & -1.0000 & 0.2202 & & 4802619 & -119.77 & 243.53 \\
\hline$(1,2,1)$ & AR1 & $\begin{array}{l}-0.1537 \\
\end{array}$ & 0.2740 & & 160.7 & -52.93 & 111.86 \\
\hline & MA1 & -1.0000 & 0.2183 & & & & \\
\hline
\end{tabular}

In the present study, ARIMA $(1,2,1)$ in ceded and ARIMA $(2,2,1)$ coastal Andhra regions) were the best fitted models through the minimum value of AIC, then used for prediction up to 10 years of the yield of groundnut in ceded districts using 15 years time series data i.e. from 2003-2004 to 2017-2018. ARIMA ( $1,2,1)$, ARIMA $(2,2,1)$ coastal Andhra regions used because the reason of its capability to make prediction using time series data with any kind of patterns and with auto correlated successive values of the time series. The study was also validated and statistically tested that the successive residuals in the fitted ARIMA $(1,2$ , 1 )ceded region, ARIMA $(2,2,1)$ coastal Andhra regions were not correlated, and the residuals appear to be normally distributed with the mean zero and constant variance. Hence,

TABLE-12 Identification of ARIMA(p,d,q) MODEL for Production in Ceded \& Coastal Andhra Regions

\begin{tabular}{|c|c|c|c|c|c|c|c|}
\hline \multicolumn{8}{|c|}{ Identification of ARIMA(p,d,q) MODEL for PRODUCTION(CEDED REGION) } \\
\hline Model & $\begin{array}{l}\text { PROD. } \\
\text { ARIMA }\end{array}$ & Coefficients & SE & Intercept & $\sigma^{\wedge 2}$ & $\begin{array}{l}\text { Log } \\
\text { likelihood }\end{array}$ & AIC \\
\hline \multirow{2}{*}{$(\mathbf{1 , 0 , 1})$} & AR1 & -0.2205 & 0.4445 & 765.2733 & \multirow[t]{2}{*}{159820} & \multirow[t]{2}{*}{-111.21} & \multirow{2}{*}{230.42} \\
\hline & MA1 & -0.1346 & 0.4025 & 74.9200 & & & \\
\hline \multirow[t]{3}{*}{$(2,1,1)$} & AR1 & -0.9458 & 0.2739 & & \multirow[t]{3}{*}{139690} & \multirow[t]{3}{*}{-103.77} & \multirow[t]{3}{*}{215.54} \\
\hline & AR2 & $\begin{array}{l}-0.6215 \\
\end{array}$ & 0.2299 & & & & \\
\hline & MA1 & $\begin{array}{l}-0.2511 \\
\end{array}$ & 0.4060 & & & & \\
\hline \multirow[t]{3}{*}{$(1,0,0)$} & AR1 & $\begin{array}{l}-0.3329 \\
\end{array}$ & 0.2349 & 765.9133 & \multirow[t]{3}{*}{161114} & \multirow[t]{3}{*}{-111.27} & \multirow[t]{3}{*}{228.53} \\
\hline & & & & 79.0824 & & & \\
\hline & MA1 & -0.7552 & 0.2130 & & & & \\
\hline \multicolumn{8}{|c|}{ Identification of ARIMA(p,d,q) MODEL for PRODUCTION(COASTAL ANDHRA REGION } \\
\hline Model & $\begin{array}{l}\text { PROD. } \\
\text { ARIMA }\end{array}$ & Coefficients & SE & Intercept & $\sigma^{\wedge 2}$ & $\begin{array}{l}\text { Log } \\
\text { likelihood }\end{array}$ & AIC \\
\hline$(1,2,0)$ & AR1 & -0.4091 & 0.2393 & & 1027 & -63.61 & 131.22 \\
\hline \multirow[t]{3}{*}{$(1,1,2)$} & AR1 & $\begin{array}{l}-0.3850 \\
\end{array}$ & 0.3058 & & \multirow[t]{3}{*}{306.1} & \multirow[t]{3}{*}{-60.87} & \multirow[t]{3}{*}{129.73} \\
\hline & MA1 & 0.4757 & 0.2873 & & & & \\
\hline & MA2 & $\begin{array}{l}-0.5243 \\
\end{array}$ & 0.2372 & & & & \\
\hline
\end{tabular}

it can be a satisfactory predictive model for the groundnut yield in ceded districts in Andhra Pradesh for the period of 2018 to 2027 . The ARIMA ( $1,2,1)$ ceded region, ARIMA $(2,2,1)$ coastal Andhra regions model projected an increment in the yield for the duration of 2018 to 2027. The prediction of 2027 is resulted approximately $\mathbf{6 2 1 6 . 1 4 8}$ ' $\mathrm{kg} / \mathrm{ha}$.(Ceded Region) \& 33374.02 'kg/ha(Coastal Andhra Region). Like any other predictive models for forecasting, ARIMA model has also limitations on accuracy of the predictions yet it is widely used for forecasting the future values for time series.

\section{Production of Groundnut Crop Conclusion}




\begin{tabular}{|l|l|l|l|l|l|l|l|}
\hline \multirow{2}{*}{$(1,2,1)$} & AR1 & -0.1537 & 0.2740 & & 160.7 & -52.93 & 111.86 \\
\cline { 2 - 5 } & MA1 & -1.0000 & 0.2183 & & & & \\
\hline \multirow{2}{*}{$(1,0,1)$} & AR1 & 0.1425 & 0.2586 & 146.7956 & 328.6 & -66.27 & 140.54 \\
\cline { 2 - 5 } & MA1 & 1.0000 & 02214 & 10.4633 & & & \\
\hline
\end{tabular}

In the present study, the ARIMA $(2,1,1)$ in Ceded Region \& ARIMA(1,2,1)in Coastal Andhra Region were the best fitted model through the minimum value of AIC, then used for prediction up to 10 years of the production of groundnut in ceded districts using 15 years time series data i.e. from 2003-2004 to 2017-2018. ARIMA $(2,1,1)$ in Ceded Region \& ARIMA(1,2,1)in Coastal Andhra Region were used because the reason of its capability to make prediction using time series data with any kind of patterns and with auto correlated successive values of the time series. The study was also validated and statistically tested that the successive residuals in the fitted ARIMA $(2,1,1)$ in Ceded Region \& ARIMA $(1,2,1)$ in Coastal Andhra Region were not correlated, and the residuals appear to be normally distributed with the mean zero and constant variance. Hence, it can be a satisfactory predictive model for the groundnut yield in ceded districts in Andhra Pradesh for the period of 2018 to 2027. The ARIMA $(2,1,1)$ in Ceded Region \& ARIMA(1,2,1)in Coastal Andhra Region models projected an increment in the production for the duration of 2018 to 2027 . The prediction of 2027 is resulted approximately $\mathbf{7 2 0 . 1 3 5 9}^{\prime} 000$ tonnes(Ceded Region) \& 163.78927'000 tonnes(Coastal Andhra Region). Like any other predictive models for forecasting, ARIMA model has also limitations on accuracy of the predictions yet it is widely used for forecasting the future values for time series. It is noticed that in Groundnut production Ceded Region is better than Coastal Andhra Region. The empirical Forecasting area, yield and production of groundnut crop: a comparative study of coastal andhra and ceded regions using- $\mathbf{R}$ findings of study could help to forecast any such commodities. The researchers and policy makers will thus get access for making further extensive research work. We firmly believe that this research has shed some important light on the subject area encompassing time series forecasts of selected agricultural crops in Seemandhra. These empirical findings can be an important source of information to many researchers and policy formulators as far as agricultural crops in Seemandhra are concerned.

\section{ACKNOWLEDGMENT}

I would like to express my heartfelt gratitude to my guide and Head of Dept. Prof. Mohammed Akhtar Palagiri who acted as a source of inspiration in all spheres of my work for giving valuable guidelines for completeing this course.

\section{REFERENCES}

[1] Madhusudana, B, "A survey on area, production and productivity of groundnut crop in India”, IOSR Journal of Economics and Finance, volume 1, PP 01-07, (2013).

[2] Muhammad Iqbal Ch, "Forecasting of wheat production: A comparative study of Pakistan And India", IJAR 4(12), 698-709, (2016).
[3] Rachana Wankhade, "Use of the ARIMA Forecasting pigeon Pea production in India ", International Review of Business and Finance, Volume 2, Number 1, pp 97-102, ( 2010).

[4] N.M.F. Rahman, "Forecasting of Boro rice production in Baangladesh: An Approach”. J.Bangladesh Agril. Univ. ; 8(1):103-112p, (2010).

[5] Najeeb Iqbal , "Forecasting wheat area and production in Pakistan", Journal of Agriculture \& Sciences, Volume.1, No.2, (2005).

[6] M.K Debnath, "Forecasting Area, production, and Yield of Cotton in India using ARIMA Model", Journal of Space Science \& Technology, Volume 2, Issue 1. (2013).

[7] M. Hemavathi , "ARIMA Model for Forecasting of Area, Production and productivity of Rice and Its Growth Status in Thanjavur District of TamilNadu”, India, International of Current Microbiology and Applied Sciences, 7(2):149-156, (2018).

[8] P.K. Sahu, "Modelling and forecasting of area, production, yield and total seeds of Rice and Wheat in SAARC Countries and the World towards Food Security", SCIEP, American Journal of Applied Mathematics and Statistics, Vol.3, No.1,34-38., (2015).

[9] Mohammed Amir Hamjah, " Rice Production Forecasting in Bangladesh: An Application of Box-Jenkins ARIMA Model”, Mathematical Theory and Modelling, Vol. 4, No 4, (2014).

[10] Muhammad, F., M., Siddiqqe, M. Bashir, S. Ahmad, “Forecasting Rice Production in Pakistan-Using ARIMA Models “, J. Of Animal Plant Sci.; 2:27-31p. (1992).

[11] Niaz Md. Farhat Rahman , “Modeling for Growth and Forecasting of pulse production in Bangladesh", Research journal of Applied Sciences, Engineering and Technology 5 (24):5578-5587. (2013).

[12] Vishwajith K..P, "Timeseries Modeling and forecasting of pulses production in India”, Journal crop and weed, 10 (2):147-154, (2014).

[13] Ashwin Darekar, "Forecasting oilseeds prices in India: Case of Groundnut”, J.Oilseeds Res..,34(4):235- 240, (2017).

[14] Bhola Nath, DS , "Forecasting Wheat production in India: An ARIMA modelling approach", Journal of Pharmacognosy and Phytochemistry, 8(1):2158-2165, (2018).

[15] Pant, D.C. and Pradeep Pal, , "Comparative Economics of Agro-processing units for Groundnut in Southern Rajasthan”, Indian Journal of Agricultural Marketing, Vol, 18, No.1, January-(2004).

[16] Ap Patel, G.N., and N.L. Agarwal, 'Price Behaviour of Groundnut in Gujarat”, Indian Journal of Marketing, Vol.7, No.2, pp.50-57, ( 1993).

[17] Mohammad Mayazzem Hossain, " Comparision of ARIMA and Neural Net Work Model to forecast the jute Production in Bangladesh", Jahingir Nagar University Journal of Science, Vol.40, pp11-18, (2017).

[18] Ansari M I and Ahmed SM," Time series analysis of tea prices: an application of ARIMA modelling and co- integration analysis", IJE,48; 49-54. (2001).

[19] Croxton.F.E., Cowden, D.J. and Klein, S, "Applied General Statistics", New Delhi: Preentice Hall of India PVT. Ltd. (1979).

[20] Pankratz, A "Forecasting with Univariate Box - Jenkins models: concepts and cases," John Wiley, New York. ,( 1983).

[21] Box, G.E.P., Jenkins, G.M. and Reinsel, G.C “ Time series analysis. Forecasting and control," Pearson Education, Delhi. , ( 1994).

[22] Makridakis, S., Wheelwright, S.C. and Hyndman, R.J, "Forecasting Methods and Applications", 3rd Edition, John Wiley, New York. ,( 1998) 
International Journal of Research in Advent Technology, Vol.7, No.11, November 2019

E-ISSN : 2321-9637

Available online at www.ijrat.org

\section{AUTHORS PROFILE}

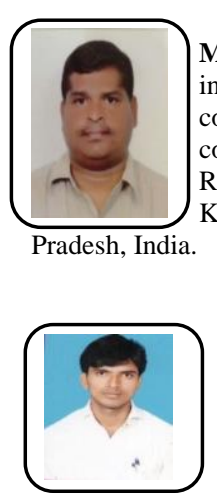

Mr. Ananda Kumar Ginka currently working as a Lecturer in Statistics in department of statistics, Sri Srinivasa Degree college, Madanapalle, Chittoor District, Andhrapradesh. He completed M.Sc., M.Ed.,M.Phil., and pursuing Ph.D

Research Scholar, Department of Statistics, Sri Krishnadevaraya University, Anantapuramu-515003, Andhra

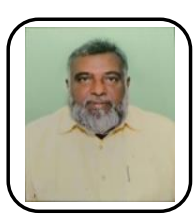

Mr. Dhanunjaya Sunkara, Has Completed M.Sc.,In Statistics And Pursuing Ph.D, Research Scholar, Department Of Statistics, Sri Krishnadevaraya University, Anantapuramu-515003, Andhra Pradesh. India.

Th Dr. Mohammed Akhtar Palagiri, Working as A Professor and Head \& Bos, Chairman, Department of Statistics, Sri Krishnadevaraya University, Anantapuramu-515003, Andhra Pradesh, India. Dr.

Mohammed Akhtar Palagiri, Has Completed M.Sc.,

M.Phil., Ph.D., In Statistics. He Has Completed 38

Research Papers in His Portfolio. 